\title{
An Alfvenic reconnecting plasmoid thruster
}

\author{
Fatima Ebrahimi $^{\oplus}, \dagger$ \\ Princeton Plasma Physics Laboratory, and Department of Astrophysical Sciences, \\ Princeton University, Princeton, NJ 08544, USA
}

(Received 29 August 2020; revised 8 November 2020; accepted 10 November 2020)

\begin{abstract}
A new concept for the generation of thrust for space propulsion is introduced. Energetic thrust is generated in the form of plasmoids (confined plasma in closed magnetic loops) when magnetic helicity (linked magnetic field lines) is injected into an annular channel. Using a novel configuration of static electric and magnetic fields, the concept utilizes a current-sheet instability to spontaneously and continuously create plasmoids via magnetic reconnection. The generated low-temperature plasma is simulated in a global annular geometry using the extended magnetohydrodynamic model. Because the system-size plasmoid is an Alfvenic outflow from the reconnection site, its thrust is proportional to the square of the magnetic field strength and does not ideally depend on the mass of the ion species of the plasma. Exhaust velocities in the range of 20 to $500 \mathrm{~km} \mathrm{~s}^{-1}$, controllable by the coil currents, are observed in the simulations.
\end{abstract}

Key words: space plasma physics, plasma devices, plasma simulation

\section{Introduction}

Natural plasma engines such as the sun continuously generate enormous magnetic energy with complex field topology, and release this magnetic energy in other forms. In the solar corona region, the linkage and the complexity of field lines, magnetic helicity, is injected through twisting field lines via shear motion of their foot points. This buildup of magnetic helicity is then released through the process of magnetic reconnection, i.e. the rearrangement of magnetic field topology of plasmas, in which magnetic energy is converted to kinetic energy and heat. On the surface of the sun, the process of magnetic helicity injection provides the reconnection sites for oppositely directed field lines to come together to reconnect and energize. In this contribution, we introduce a novel thruster concept, which takes advantage of a similar effect to convert magnetic energy to kinetic energy to produce thrust. In this concept, the reconnection sites are also generated via helicity injection, but by driving current along open field lines rather than twisting them via shear motion. This concept is based on the combination of two key physical effects: (i) magnetic helicity injection and (ii) axisymmetric magnetic reconnection. Significant thrust is generated in the form of plasmoids (confined plasma objects in closed magnetic loops) when helicity is injected into a cylindrical vessel to induce magnetic reconnection. Existing space-proven plasma thrusters, including the ion thruster (Stuhlinger 1964; Choueiri 2009) and the Hall-effect thruster (Morozov et al. 1972;

$†$ Email address for correspondence: ebrahimi@pppl.gov 
Raitses, Smirnov \& Fisch 2007), electrostatically accelerate ions to exhaust velocities $v_{e}$ of tens of kilometres per second to produce thrust. However, for space exploration to Mars and beyond, high-thrust electromagnetic propulsion with exhaust velocities of tens to hundreds of kilometres per second is needed. This new concept, capable of reaching high and variable exhaust velocities, could complement existing designs for such missions.

For efficient use of propellant and propulsion power during space travel, thrusters should have an exhaust velocity similar to the velocity difference $\Delta v$ between the origin and destination celestial bodies. This is quantitatively expressed by the Tsiolkovsky rocket equation

$$
\Delta v=v_{e} \ln \left(m_{0} / m_{1}\right),
$$

where $m_{0}$ and $m_{1}$ are the total mass, including propellant, at the origin and destination, respectively. Equation (1.1) shows that for a given $v_{e}$ and final mass $m_{1}$ a linear increase in $\Delta v$ requires an exponential increase in initial mass $m_{0}$. If the propellant is fully spent at the destination, the ratio $\left(m_{0}-m_{1}\right) / m_{0}$ is the propellant mass ratio. For conventional chemical thrusters (rockets), the exhaust velocity is limited by the speed of chemical reactions to about $1-4 \mathrm{~km} \mathrm{~s}^{-1}$ (or specific impulse $I_{\mathrm{sp}}$ between 100 and $400 \mathrm{~s}$, where $I_{\mathrm{sp}}=v_{e} / g_{0}$, in which $g_{0}=9.8 \mathrm{~m} \mathrm{~s}^{-2}$ is the standard gravity). Conventional rockets are therefore efficient only for space missions that can be performed with a $\Delta v$ budget of about $4 \mathrm{~km} \mathrm{~s}^{-1}$, e.g. a mission from low Earth orbit to low Moon orbit. Even for a highly optimized mission from low Earth orbit to Mars, lasting 3-5 months and with a brief launch window every 2-3 years, a $\Delta v=6 \mathrm{~km} \mathrm{~s}^{-1}$ is needed. With an optimistic assumption of $v_{e}=4 \mathrm{~km} \mathrm{~s}^{-1}$, (1.1) gives a propellant mass ratio of $78 \%$, i.e. on launch from low Earth orbit more than three-quarters of the mass is propellant. Thus only Earth's immediate neighbours in our solar system are within reach of conventional rockets.

To surpass the exhaust velocity allowed by limited chemical energy density and reaction rates, electromagnetic propulsion can be used (Goebel \& Katz 2008; Dale, Jorns \& Gallimore 2020). Existing space-proven plasma thrusters can reach a specific impulse $I_{\mathrm{sp}}$ of about a couple of thousands of seconds (i.e. $v_{e}$ of about tens of kilometres per second). High-thrust electromagnetic propulsion with $I_{\text {sp }}$ of tens of thousands of seconds is needed to explore the solar system beyond the Moon and Mars, as well as to rendezvous with asteroids, to deflect them if they are on a collision course with Earth, or to capture them for use as a source of water and construction materials to support human presence in space. The unique feature of the plasmoid thruster introduced here is its high and variable $I_{\mathrm{sp}}$, in the range 1000 to $50000 \mathrm{~s}$, which would be a key advantage for space missions with a large $\Delta v$, i.e. to Mars and beyond. Here, we show that these high specific impulses could be achieved through continuous production of plasmoids to accelerate ions via a magnetic reconnection process.

Magnetic reconnection, which is ubiquitous in natural plasmas, energizes many astrophysical settings throughout our solar system including the solar corona (solar flares), solar wind, planetary interiors and magnetospheres (see Ji et al. (2020) and references therein), as well as throughout our universe, such as flares from accretion discs around supermassive black holes (Ripperda, Bacchini \& Philippov 2020). Magnetic reconnection causes particle acceleration to high energies, heating, energy and momentum transport (Ebrahimi \& Prager 2011) and self-organization. The Parker Solar Probe (Fox et al. 2016) also provides access to a new frontier for exploring and providing observational evidence of large- and small-scale reconnecting structures in the solar corona. In laboratory fusion plasmas, plasmoid-mediated reconnection has been shown to be important during plasma start-up formation (Ebrahimi \& Raman 2015), nonlinear 
growth of an internal kink mode (Biskamp 1986; Günter et al. 2015) as well as transient explosive events such as edge localized modes in tokamaks (Ebrahimi 2017). Here, we demonstrate a practical application of plasmoid-mediated reconnection, namely for space propulsion.

The new type of plasma thruster we are here proposing uses an innovative magnetic configuration to inject magnetic helicity using two annular electrodes biased by a voltage source, thereby inducing spontaneous reconnection via formation of a current sheet, which continuously breaks and generates plasmoids. The concept of biasing open field lines to stretch lines of force and form 'plasma rings' (Alfven, Lindberg \& Mitlid 1960) was first introduced in the so-called coaxial plasma gun (accelerator) experiments in 1960 (Alfven et al. 1960; Marshall 1960). Since then, coaxial (annular) plasma accelerators have been extensively used and evolved for various applications, including for fusion plasmas to form spheromaks (Jarboe et al. 1983; McLean et al. 2002; Hsu \& Bellan 2003) and to fuel tokamaks with compact toroids (Hammer et al. 1988; Brown \& Bellan 1990; Raman et al. 1994). The plasma accelerator has also been proposed as a magnetoplasmadynamic thruster for propulsion applications (Cheng 1971; Schoenberg et al. 1993) and for generating high-velocity plasma jets (Witherspoon et al. 2009). In all these annular plasma accelerators the Lorentz $\boldsymbol{J} \times \boldsymbol{B}$ force generated by a self-induced magnetic field accelerates plasmas to large velocities. In our new concept the acceleration is instead due to magnetic reconnection (Zweibel \& Yamada 2009; Yoo et al. 2017). Unlike existing plasma accelerators, the thrust is generated from the acceleration of bulk fluid due to continuous formation of reconnecting plasmoids in the magnetohydrodynamic (MHD) regime. Neither external pulsing nor rotating fields (Chesny et al. 2017; Bathgate et al. 2018) are required here for acceleration through reconnection.

Axisymmetric reconnecting plasmoids are secondary magnetic islands, which are formed due to plasmoid instability. At high Lundquist number, the elongated current sheet becomes MHD unstable due to the plasmoid instability (Biskamp 1986; Tajima \& Shibata 1997; Loureiro, Schekochihin \& Cowley 2007; Bhattacharjee et al. 2009; Daughton et al. 2009; Ebrahimi \& Raman 2015; Comisso et al. 2016), an example of spontaneous reconnection. The transition to plasmoid instability was shown to occur when the local Lundquist number $S=L V_{A} / \eta$ ( $V_{A}$ is the Alfven velocity based on the poloidal reconnecting magnetic field, $L$ is the current sheet length and $\eta$ is the magnetic diffusivity) exceeds a critical value (typically a few thousand). Our thruster concept is based on the formation of this elongated current sheet for triggering fast reconnection and plasmoid formation. Effects beyond MHD may also contribute to fast reconnection as the current sheet width $\left(\delta_{\mathrm{sp}}\right)$ becomes smaller than the two-fluid or kinetic scales (Cassak, Shay \& Drake 2005; Ji \& Daughton 2011). However, for thruster application we desire system-size MHD plasmoid formation (with radius ranging from a few to tens of centimetres), where kinetic effects become subdominant for low-temperature plasma (in the range of a few eV to a couple of tens of $\mathrm{eV}$ ). Here, the MHD plasmoid-mediated reconnection occurs at high Lundquist number (about $10^{4}$ and above), which is achieved at high magnetic field rather than low magnetic diffusivity (or high temperature). To form a single or multiple X-point reconnection site, oppositely directed biased magnetic field (in the range of 20-1000 G) is injected through a narrow gap in an annular device. We find that the plasmoid structures demonstrated in resistive (or extended) MHD simulations produce high exhaust velocity and thrust that scale favourably with applied magnetic field. It will be shown that the fluid-like magnetic plasmoid loops continuously depart the magnetic configuration about every $10 \mu \mathrm{s}$ with Alfvenic velocities in the range of 20 to $500 \mathrm{~km} \mathrm{~s}^{-1}$, and the thrust does not ideally depend on the mass of the ion species of the plasma. 
(a)

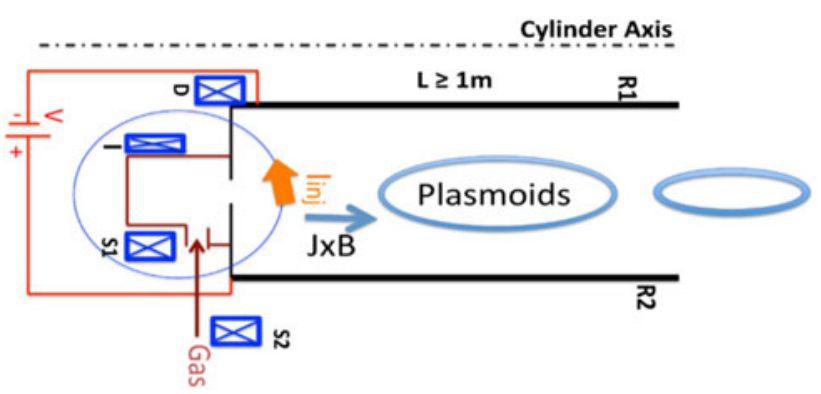

(b)

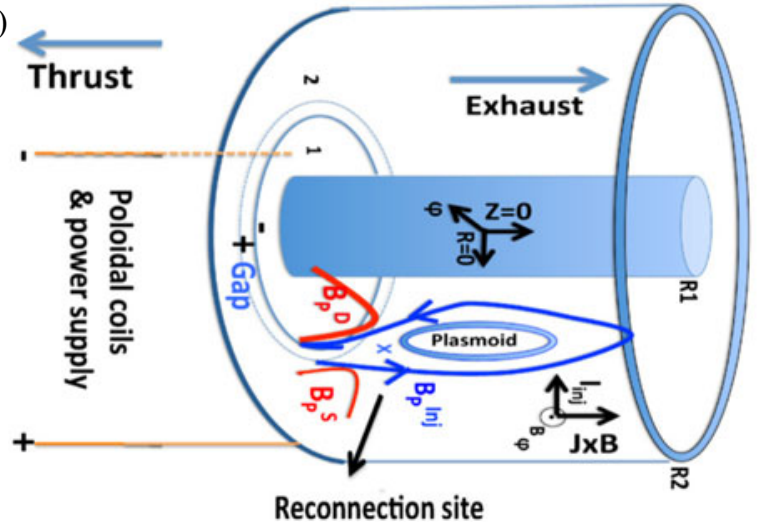

FIGURE 1. A schematic of $(a)$ the vertical cross-section and $(b)$ the entire domain of the reconnecting plasmoid thruster. In an annular configuration, injected poloidal field $B_{P}^{\text {inj }}$ (blue circle) is generated by poloidal field injector coil $(I)$, while current $\left(I_{\mathrm{inj}}\right)$ is pulled along open

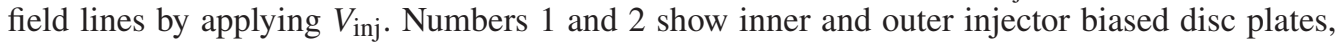
respectively, separated by the injector gap. All the axisymmetric poloidal coils $(I, D, S 1, S 2)$ are located to the left of these plates. For formation of an elongated current sheet to induce spontaneous reconnection, the detachment coil $D$ and shaping coils $S 1$ and $S 2$ are also energized to generate the poloidal fields $B_{P}^{D}$ and $B_{P}^{S}$ (shown in red in $(b)$ ).

\section{Schematics of the thruster}

Figure 1 shows the main parts of the reconnecting plasmoid thruster in an annular configuration. Magnetic helicity injection starts with an initial injector poloidal field $\left(B_{P}^{\mathrm{inj}}\right.$, in blue, with radial, $R$, and vertical, $Z$, components), connecting the inner and outer biased plates in the injector region. Gas is injected and partially ionized by applying an injector voltage $V_{\text {inj }}$ of a few hundred volts between the inner and outer plates (indicated by numbers 1 and 2), which also drives a current $I_{\text {inj }}$ along the open magnetic field lines. Plasma and open field lines expand into the vessel when the Lorentz force $J_{\text {pol }} \times B_{\phi}$ exceeds the field line tension of the injector poloidal field. The azimuthal $(\phi)$ field shown here, $B_{\phi}$, is generated through injector current $\left(I_{\text {inj }}\right)$ alone (by applying $V_{\text {inj }}$ ), or can be provided externally. Plasma formation through electron impact ionization has been widely used by plasma accelerators and other helicity injection experiments (Jarboe et al. 1983; Hammer et al. 1988; Brown \& Bellan 1990; Raman et al. 1994; McLean et al. 2002; Hsu \& Bellan 2003; Raman et al. 2003; Witherspoon et al. 2009; Raman et al. 2011). The conventional Townsend avalanche breakdown theory is applicable for coaxial helicity injection experiments (Hammond, Raman \& Volpe 2017; R. Raman, 
Private communication on helicity injection experiments on HIT-II and NSTX, 2020), a configuration similar to the thruster proposed here.

Up to this point the concept of magnetic helicity injection through the linkage of the injected poloidal field and injected azimuthal field from poloidal current along the open field lines is similar to that of conventional annular accelerators. However, at this stage we introduce the new concept of plasmoid-mediated reconnection for generating thrust, i.e. through forming a vertically elongated (along $Z$ ) azimuthal current sheet $\left(J_{\phi}\right)$, which contributes to the Lorentz force. To continuously form a current sheet at the reconnection site, the detachment and shaping poloidal fields $B_{P}^{D}$ and $B_{P}^{S}$ (shown in figure $1 b$ and produced by the $D, S 1$ and $S 2$ coils) are utilized and have an instrumental role for this thruster concept. These coils can be effectively used to strongly and radially squeeze the injector poloidal field to cause oppositely directed field lines in the $Z$ direction (shown in blue arrows at the reconnection site) to reconnect. To form this reconnection site, the currents in the detachment and shaping coils are in the opposite direction to the current in the injector coil, and the detachment-coil current is of magnitude equal to or larger than the injector-coil current. As a result, azimuthally symmetric system-sized plasmoid structures are detached and ejected to produce thrust.

\section{Global extended MHD simulations}

We perform time-dependent extended MHD simulations of the thruster using the NIMROD code (Sovinec et al. 2004), which is a community code supported by DOE, and has been extensively used and validated for various helicity injection fusion experiments (Bayliss, Sovinec \& Redd 2011; Hooper et al. 2012; Morgan, Jarboe \& Akcay 2019), including start-up helicity injection experiments for spherical tokamaks (NSTX and NSTX-U) (Ebrahimi et al. 2013, 2014; Hooper et al. 2013; Ebrahimi \& Raman 2016). We model coil currents that produce the needed injected field for the reconnection site. Simulations are performed for a constant-temperature model (pressure is not evolved in time) with constant-in-time poloidal-field coil currents. We have optimized and varied extensively the current in the poloidal coils $(I, D, S 1, S 2)$ to form a reconnection site and a current sheet. Our extended MHD model consists of combined Faraday and generalized Ohm's laws and the momentum equation:

$$
\begin{gathered}
\rho\left(\frac{\partial \boldsymbol{V}}{\partial t}+\boldsymbol{V} \cdot \nabla \boldsymbol{V}\right)=\boldsymbol{J} \times \boldsymbol{B}-\nabla \cdot \Pi, \\
\frac{\partial \boldsymbol{B}}{\partial t}=-\boldsymbol{\nabla} \times\left(-\boldsymbol{V} \times \boldsymbol{B}+\eta \boldsymbol{J}+\frac{1}{n e} \boldsymbol{J} \times \boldsymbol{B}+\frac{m_{e}}{n e^{2}} \frac{\partial \boldsymbol{J}}{\partial t}\right),
\end{gathered}
$$

where $V$ is the centre-of-mass velocity and $\rho$ is the mass density of a plasma with magnetic field $\boldsymbol{B}$ and current density $\boldsymbol{J}$. The stress tensor $(\Pi)$ is treated as $-\rho \nu \nabla^{2} \boldsymbol{V}$ or $-\rho v W$, where $v$ is the kinematic viscosity and $W$ is the rate of strain tensor. In all simulations, the kinematic viscosity is chosen to give Prandtl number $P m=\eta / v=2-7.5$. The magnetic diffusivities used in the simulations $\left(\eta=8-32 \mathrm{~m}^{2} \mathrm{~s}^{-1}\right)$ are equivalent to constant low temperatures of $T_{e} \approx 5-14 \mathrm{eV}$, according to the Spitzer resistivity relation $\left(\eta\left(\mathrm{m}^{2} \mathrm{~s}^{-1}\right)=410 T^{-3 / 2}(\mathrm{eV})\right)$. A constant electric field is applied across the narrow injector gap (located between the two injector plates $R=0.54-0.57 \mathrm{~m}$, shown in figure 2). Perfectly conducting boundary conditions with no slip are used, except at the injector gap, which has a normal $E \times B$ flow, where a constant-in-time electric field is applied (Ebrahimi et al. 2013; Hooper et al. 2013; Ebrahimi 2016). We use a poloidal grid with $45 \times 90$ sixth-order finite elements in a global $(R, Z)$ geometry and azimuthal mode numbers $\left(n_{\phi}\right)$ up to 22 modes. A uniform number density $(n)$ of $4 \times 10^{18} \mathrm{~m}^{-3}$ for a 


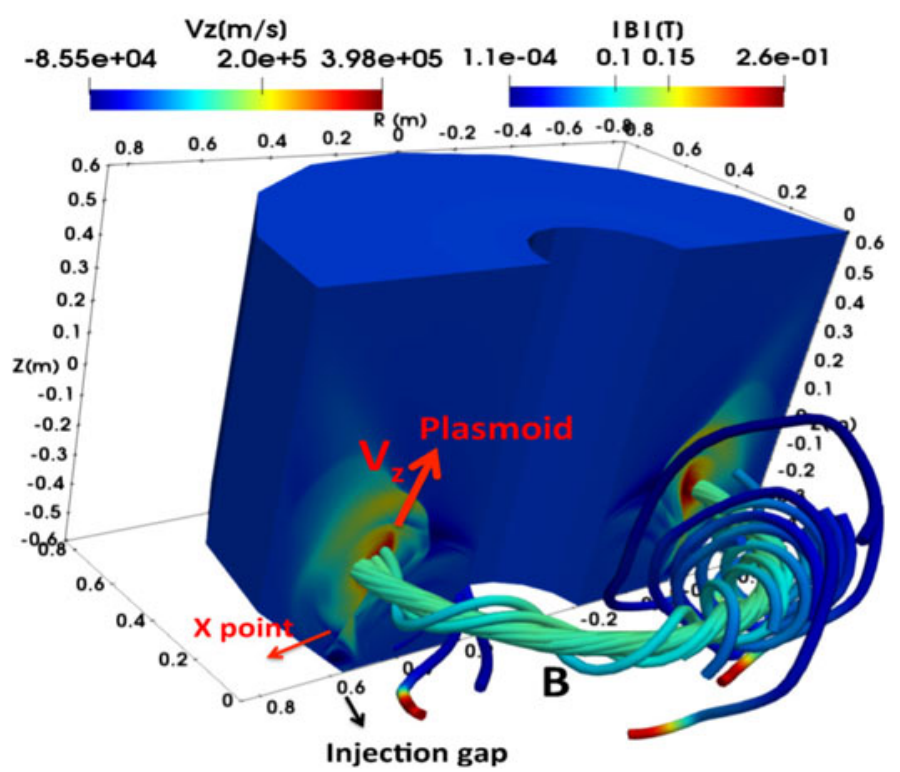

FIGURE 2. The formation of momentum-carrying plasmoid during three-dimensional global extended (two-fluid) MHD simulations. The computational domain and the poloidal coil configurations are the same as the schematics in figure 1. Plasmoid ion (helium) velocity $V_{z}$ is seen in the poloidal $(R-Z)$ cross-section. The velocity structure remains azimuthally symmetric. Following of the magnetic field line shows a closed magnetic loop associated with the plasmoid formation during reconnection.

deuterium or helium plasma is used. Simulations are performed with various coil currents in a straight plasma domain configuration, shown in figure 1, in a thruster channel with inner and outer radii of $R_{1}=0.21 \mathrm{~m}$ and $R_{2}=0.85 \mathrm{~m}$, with the injector plates ( 1 and 2, shown in figure 1) located at $Z=-0.8 \mathrm{~m}$. In general the results do not vary with the axial length of the thruster within the range $1-2 \mathrm{~m}$ investigated, and with the angle of the lower injector plates with the side walls $\left(90^{\circ}\right.$ and $\left.145^{\circ}\right)$. The locations of the coils are adjusted for simulations performed in different domain sizes. In the simulations shown in figures 3 and 4 , the injector $(I)$, detachment $(D)$ and shaping field $(S 1, S 2)$ coils are located at $R=0.52,0.31,0.76,1 \mathrm{~m}$ and $Z=-1,-0.82,-1.05,-0.81 \mathrm{~m}$, respectively. Fields $B_{P}^{D}$ and $B_{P}^{S}$ are static and assumed to have penetrated through the bounding surfaces.

A cut of the general computational domain of annular geometry is shown in figure 2 . In this simulation we have used a two-fluid model, i.e. using both the Hall term and electron inertia terms in equation (3.2) with an increased electron mass $\left(m_{i} / m_{e}=73\right)$. The exhaust vertical velocity of azimuthally symmetric plasmoids reaches about $400 \mathrm{~km} \mathrm{~s}^{-1}$ (figure 2). Most of the momentum is along the large plasmoid (and current sheet). The Alfvenic-type outflows obtained here $\left(V_{A}\right.$ for this case using $B_{z}=0.08$ is about $\left.460 \mathrm{~km} \mathrm{~s}^{-1}\right)$ are due to spontaneous reconnection and the plasmoid ejection. In the two-fluid model, fast reconnection is caused by the Hall current, which is a signature of the decoupling of electron and ion motions at scales below the ion skin depth $d_{i}\left(d_{i}=c / \omega_{p i}\right.$, where $c$ is the speed of light and $\omega_{p i}$ is the ion plasma frequency). Here $d_{i}$ is calculated to be relatively large, about $11 \mathrm{~cm}$. Current density and axial velocity for the two-fluid simulation at two times are also shown in figure 3 . The formation of the single $X$-point during Hall reconnection at $t_{1}$ and then the subsequent ejection of a large plasmoid at $t_{2}$ with large exhaust velocity of $420 \mathrm{~km} \mathrm{~s}^{-1}$ are observed. The two-fluid results in terms of 
(a) $\mathrm{t}_{1}=0.0385 \mathrm{~ms}$
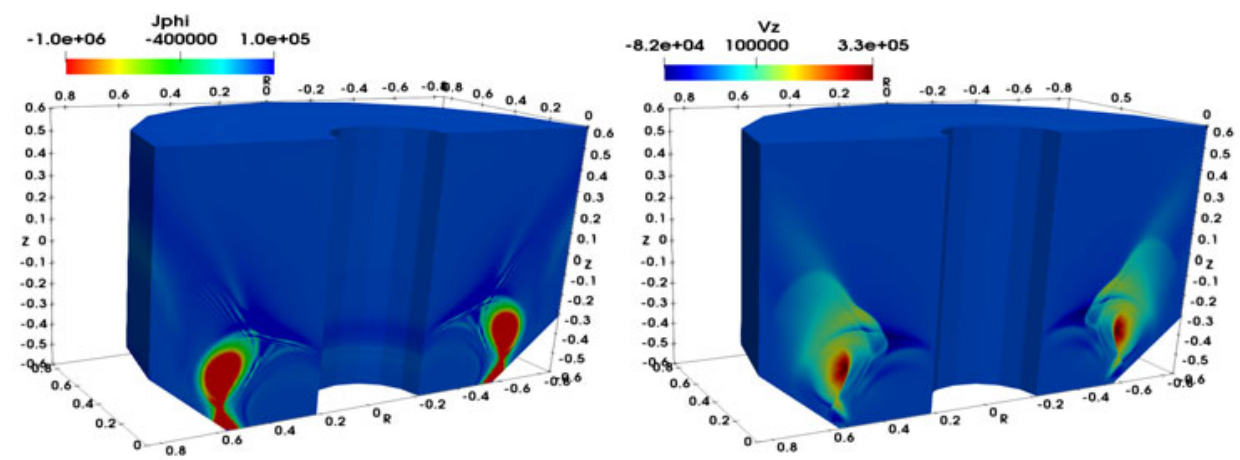

(b)

$\mathrm{t}_{2}=0.0395 \mathrm{~ms}$
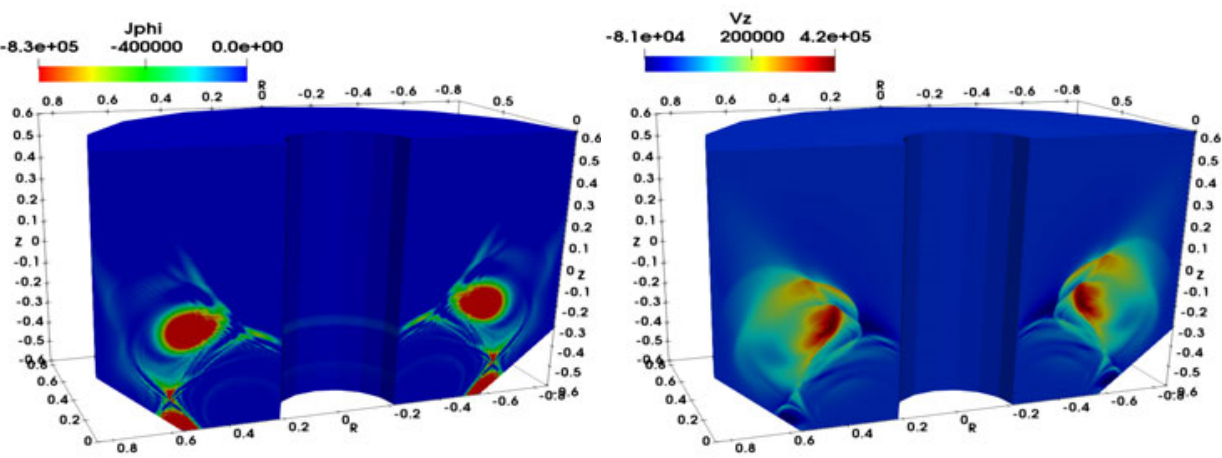

FIGURE 3. Current density $\left(\mathrm{A} \mathrm{m}^{-2}\right)$ and axial velocity $\left(\mathrm{m} \mathrm{s}^{-1}\right)$ for the same two-fluid simulation shown in figure 2 , at the two times $(a) t_{1}=0.0385$ and at $1 \mu \mathrm{s}$ later at $(b) t_{2}=0.0395$.

exhaust speed of plasmoids during reconnection are similar to the resistive MHD model (shown below). We therefore present below more detailed results from resistive MHD simulations to focus on the plasmoid-mediated reconnection for this concept.

For this thruster concept, we start with an initial injector poloidal field $\left(B_{P}^{\mathrm{inj}}\right)$ with a very narrow footprint (where open field lines intersect the inner and outer plates) to form a reconnection site. Poloidal $R-Z$ cuts of the oppositely directed injected reconnecting field $\left(B_{z}\right)$, which provides the primary reconnection site, and the azimuthal field, which is intrinsically generated by the poloidal injector current in the injection region, are shown in figures 4(a) and 4(b), respectively. As the injector voltage is applied (by ramping $V_{\text {inj }}$ from zero to about $200 \mathrm{~V}$ ), the generated azimuthal field could reach as high as $2000 \mathrm{G}$ (figure $4 b$ ), which causes the injector poloidal field to start expanding in the thruster channel. As the field is expanded in the domain, the static fields $B_{P}^{D}$ and $B_{P}^{S}$ radially pinch the injector field around the injector gap to form a primary exhaust reconnecting current sheet, as shown in blue (figure $4 c$ ). The plasmoid instability is here triggered at local Lundquist number $S \sim 12000$ (based on $B_{z} \sim 500 \mathrm{G}, L=0.5 \mathrm{~m}$ and $\eta=16 \mathrm{~m}^{2} \mathrm{~s}^{-1}$ ). The formation of a plasmoid along the current sheet is seen in figure $4(c, d)$ (in blue). For comparison, the exhaust current density for the two-fluid simulations (shown in figure 2) with single $X$-point Hall reconnection topology (Huang, Bhattacharjee \& Sullivan 2011) is 

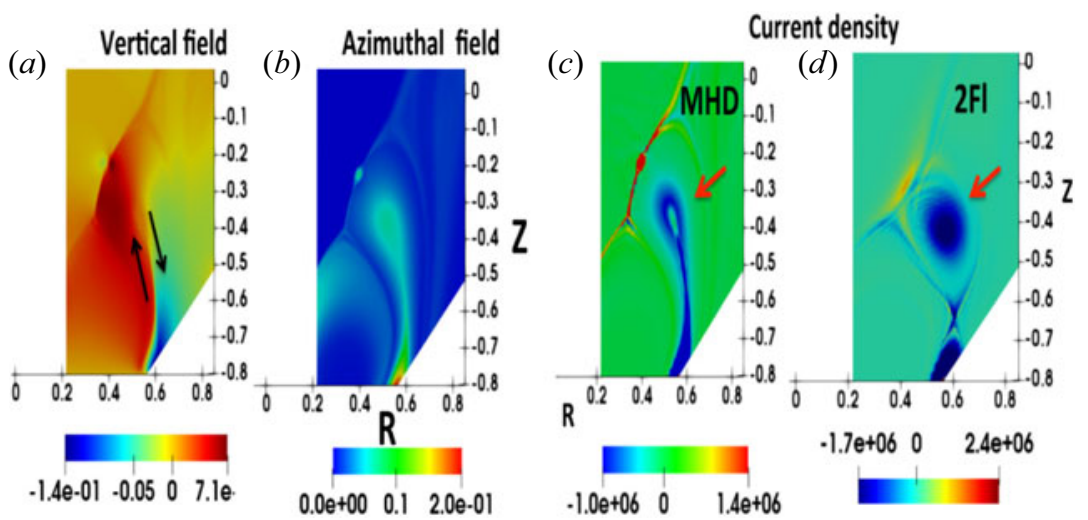

FIGURE 4. Poloidal $R-Z$ cut of $(a)$ injected vertical magnetic field $B_{z}(\mathrm{~T})$ and $(b)$ generated azimuthal field $B_{\phi}(\mathrm{T})$ at $t=0.044 \mathrm{~ms}$ during the helicity injection (black arrows show the oppositely directed reconnecting $B_{z}$ field at the reconnection site). Azimuthal current density $J_{\phi}\left(\mathrm{A} \mathrm{m}^{-2}\right)$ with $(c)$ MHD and $(d)$ two-fluid model (from same simulation as results in figure 2 ). Red arrows show reconnecting plasmoids.

shown in figure $4(d)$. We have also performed two-fluid simulations at smaller $d_{i}$ (of about $2 \mathrm{~cm}$ at higher density), and then recover the elongated single-fluid current sheet.

The formation of a large plasmoid with exhaust outflow that reaches as high as $V_{z}=400 \mathrm{~km} \mathrm{~s}^{-1}$ around the reconnection region at later time $t=0.046 \mathrm{~ms}$ is shown in figure 5. The maximum exhaust velocity is mainly along the current sheet and the plasmoid (as can be seen in figure $5 a, b)$ and exceeds the visco-resistive outflows of the MHD Sweet-Parker (S-P) model, $V_{\text {out }} / V_{A(\mathrm{pol})}=1 / \sqrt{1+P m} \sim 170 \mathrm{~km} \mathrm{~s}^{-1}$ (where $\left.V_{A(\mathrm{pol})}=B_{\text {in }(z)} / \sqrt{\mu_{0} \rho}\right)$. The continuous spontaneous breaking of the current sheet and the subsequent formation of current-carrying magnetically self-confined loops, i.e. plasmoids, occur at later times (figure $5 b, c$ ), as long as the voltage is applied. As seen from the Poincaré plot in figure 5(c), two large plasmoids are formed. At this later time, as the first plasmoid is already ejected, the open field lines start to close again and a large-volume closed field line in the form of a second plasmoid is formed and departs the device with a high outflow velocity of about $460 \mathrm{~km} \mathrm{~s}^{-1}$ (figure $5 b$ ). The maximum outflows are both along the exhaust current sheets (shown in blue in figure $5 d, e$ ) in the form of outflow jets, as well as along the plasmoids. We should note that small-scale plasmoids are also formed along a current sheet (seen in red colour in figure $4 c$ ) above the primary exhaust current sheet. Although these also contribute to positive large vertical outflows, they are less important, as they are not directly connected to the injector plates. It is important to note that although all the simulations above are three-dimensional (by including 21 non-axisymmetric $\left(n_{\phi} \neq 0\right)$ modes), all the structures during nonlinear evolution shown in figures $2-5$ remain azimuthally symmetric (axisymmetric). In these simulations, the time scale for the cyclic ejection of large-scale axisymmetric plasmoids is around $10 \mu \mathrm{s}(4-5$ Alfven transit times). This time scale is much shorter for any non-axisymmetic disturbance to growth to large amplitudes.

\section{Scaling of the exhaust velocity with reconnecting field}

To further examine the variation and the dependence of the exhaust velocity on the injected field $\left(B_{z}\right)$, we have performed simulations with two different magnetic configurations in which we have varied the coil currents. We should note that in all the 

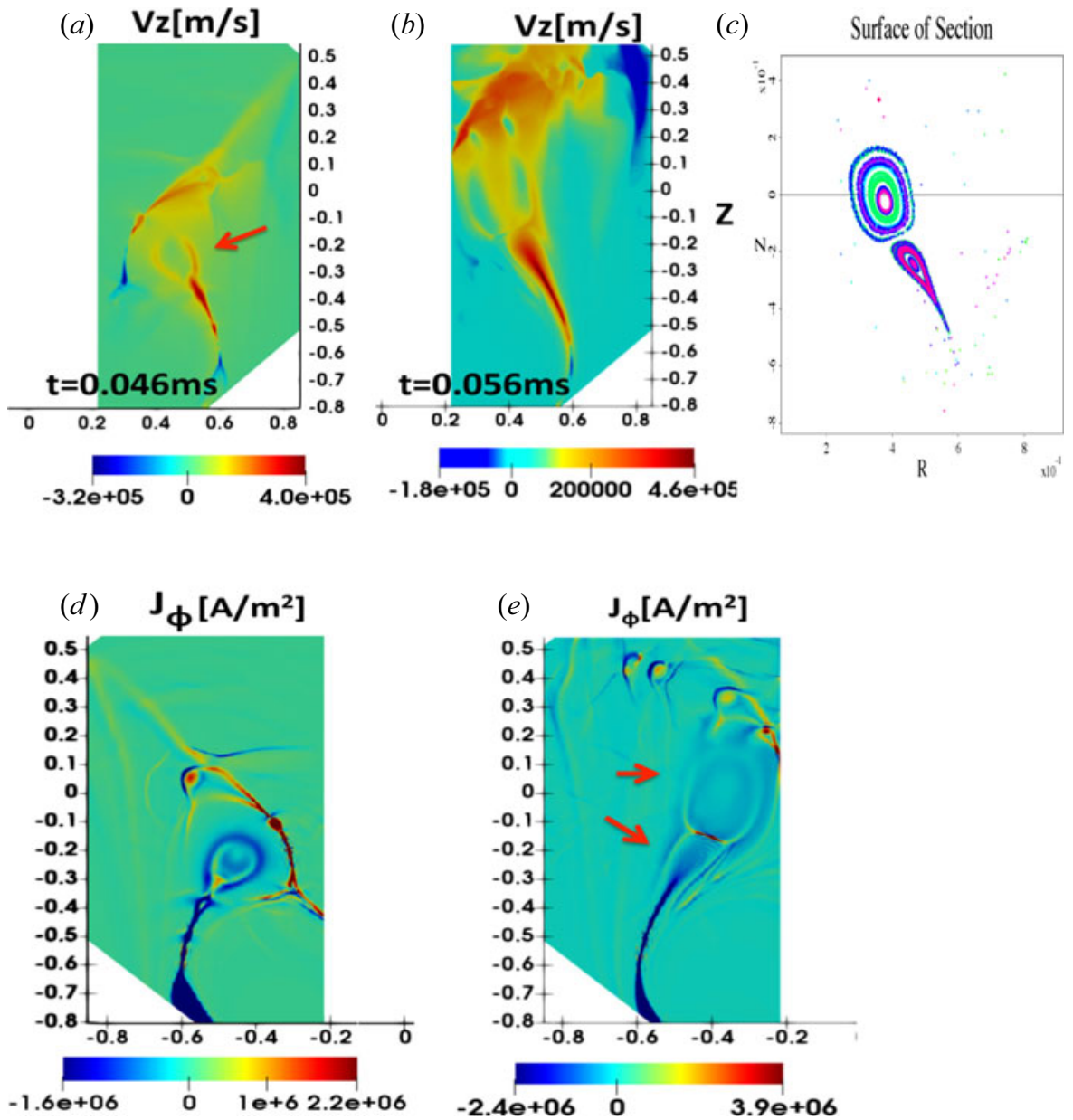

Figure 5. Poloidal $(R-Z)$ cuts of vertical flow velocity at $(a) t=0.046 \mathrm{~ms}$ and (b) $t=0.056 \mathrm{~ms}$; (c) Poincaré plot. Plasmoid ejection is shown by the red arrow. Axisymmetric azimuthal current densities at $(d) t=0.046 \mathrm{~ms}$, and $(e) t=0.056 \mathrm{~ms}$.

simulations presented above the azimuthal field is intrinsically generated. Here we also use an external azimuthal guide field of about $7000 \mathrm{G}$, which would not affect the scaling, as we only use the reconnecting $B_{z}$ field in figure 6 . The first configuration is shown in figure 6 in blue triangles at $B_{\text {rec }}=400 \mathrm{G}$ (case 1), which results in $V_{z}=300 \mathrm{~km} \mathrm{~s}^{-1}$. To scan over $B_{\text {rec }}$, we first reduce the detachment coil current (which gives $B_{z}=200 \mathrm{G}$, case 2), and then we reduce the current in all the coils by $50 \%$ (giving $B_{z}=130 \mathrm{G}$, case 3 ), and much reduced outflow velocity of about $60 \mathrm{~km} \mathrm{~s}^{-1}$. In the second configuration, we use a much higher ratio of the two shaping coil currents, but with the same injector and detachment coil currents as the first configuration. The dependency of the outflow on $B_{\text {rec }}$ for the cases in this configuration is also shown (by red diamonds) in figure 6. We also compare the outflows (maximum flows along the exhaust current sheet) obtained in the simulations with the slow S-P type reconnection model outflows in a visco-resistive MHD plasma (with $P m=7.5)$. Using $B_{z}$ from the simulations, figure 6 shows the calculated outflows based on the local S-P model (black triangles) versus $B_{z}$. It is shown that the outflows from MHD simulations do favourably scale with $B_{z}$, are higher (more than double) compared to the 


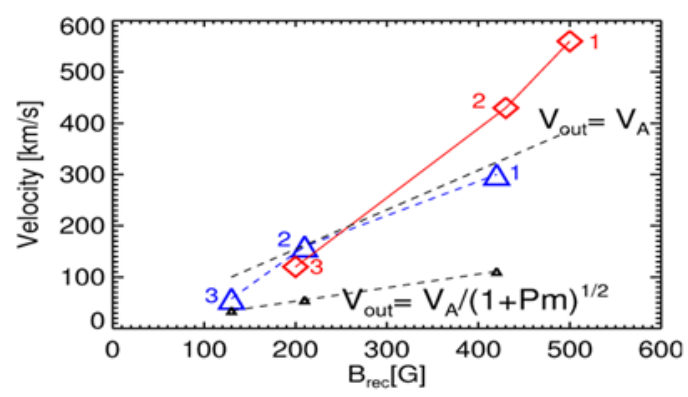

FIGURE 6. Maximum exhaust velocity obtained from simulations versus reconnecting magnetic field for two magnetic configurations with different ratio of poloidal coil currents. The dashed black line with triangles shows the theoretical S-P scaling.

slow $\mathrm{S}-\mathrm{P}$ velocities and are due to the fast plasmoid-mediated reconnection. It is therefore found that the exhaust velocities in these magnetic configurations are Alfvenic-type reconnecting outflows as they are strongly associated with the strength of $B_{\text {rec }}$.

\section{Thrust and the thrust to power ratio}

Because the plasmoids are ejected at the Alfven velocity, the expression for the thrust (Lovberg 1971) becomes $F=\rho V_{A}^{2} A$, where $A$ is the area of the plasmoid cross-section. Notably, the thrust then does not depend on $\rho$, and it scales as the magnetic field squared $\left(B^{2}\right)$. For example, for plasmoids with radius $10 \mathrm{~cm}$ (as in figure $3 d$ ) and reconnecting field of $B=800 \mathrm{G}$, the calculated thrust is about $50 \mathrm{~N}$, taking into account a duty cycle of about $33 \%$ (i.e. the distance between two consecutive plasmoids is twice the plasmoid length). The input power is given by $P_{\text {inj }}=I_{\text {inj }} V_{\text {inj }}$, where $I_{\text {inj }}=2 \pi r B_{\phi} / \mu_{0}$. In general $I_{\text {inj }}$ could vary from a few to a few hundred kiloamps. In our simulations, $I_{\text {inj }}$ is about $100 \mathrm{kA}$ (equivalent to $B_{\phi} \sim 500 \mathrm{G}$ ), corresponding to about $10 \mathrm{MW}$ of power. For this unoptimized high-power case (with a thrust of 50-100 N), the ratio of thrust over power is thus about $5-10 \mathrm{mN} \mathrm{kW}^{-1}$. We have not yet performed a systematic optimization, but tentatively the optimal parameter range for this new thruster will be $I_{\mathrm{SP}}$ (specific impulse) from 2000 to $50000 \mathrm{~s}$, power from 0.1 to $10 \mathrm{MW}$ and thrust from 1 to $100 \mathrm{~N}$. It would thus occupy a complementary part of parameter space with little overlap with existing thrusters.

In helicity injection start-up plasma experiments (with an injection region similar to here), plasma has been efficiently produced, and both plasma and magnetic fields have been successfully injected via an injector gap (Raman et al. 2003, 2011). The fundamentals of plasma production and ionization for this concept are essentially the same as for an unmagnetized direct-current gas discharge. As shown by Hammond et al. (2017), for keeping the operating voltage in a reasonable range of a few hundred volts (for acceptable cathode sputtering and good ionization efficiency), the Paschen curve imposes a minimum gas pressure. For example, for our application the connection length $\left(L_{c}\right)$ is about $10 \mathrm{~cm}$ (depending on the vertical and azimuthal magnetic fields), which requires a gas pressure of tens of millitorrs (we used $L_{c} P$ of about 6 Torr $\times$ mm given by Hammond et al. (2017), for an operating point reasonably close to the Paschen minimum). Operating voltages from a few hundred up to a thousand volts have routinely been used for helicity injection experiments, including plasma accelerators as well as plasma start-up for current drive. Significant cathode erosion (from sputtering or arcing) in the injector region has not been reported. For long-pulse operation, the cathode is sometimes coated with graphite 
or tungsten to minimize sputtering (in Raman et al. (2003) and in other helicity injection experiments). Once the plasmoid has formed, the simulations show that it stays away from the walls and should therefore not contribute to wall erosion. In the simulations walls provide the necessary boundary conditions in the domain; however, more evolved versions of this thruster might in fact be wall-less. The details of neutral dynamics also remain for future work.

\section{Summary}

Here, we have presented a new concept for the generation of thrust for space propulsion. With a low plasma temperature of only a few eV, the plasmoid objects, which could have diameters as large as several tens of centimetres, are generated in a fluid-like (MHD and two-fluid Hall) regime and move with the centre of mass of the plasma. The concept is explored via three-dimensional extended MHD simulations of reconnecting plasmoid formation during helicity injection into an annular channel. Based on the simulations, we find that there are fundamentally several advantages of this novel thruster, including the following. (i) There is a high and variable exhaust velocity as large as $500 \mathrm{~km} \mathrm{~s}^{-1}$ with injected poloidal field of 500-600 G. (ii) There is large and scalable thrust - depending on the size of plasmoid and magnetic field strength, the thrust can range at least from a tenth of a newton to tens of newtons. As the reconnecting plasmoids leave the device at the Alfven velocity, the thrust scales as magnetic field squared. (iii) The thrust does not ideally depend on ion mass, so plasma can be created from a wide range of gases, including gases extracted from asteroids. We should note that the reconnection process is advantageous for space propulsion, as the detachment from the magnetic field in the nozzle (Arefiev \& Breizman 2005) is not an issue here. Plasmoids are closed magnetic structures; they are detached from the moment they are created.

Lastly, the experimental NSTX camera images during helicity injection plasma start-up in Ebrahimi \& Raman (2015) (and the supplementary movie there), which show distinct plasmoids leaving the device with velocities of about $25 \mathrm{~km} \mathrm{~s}^{-1}$, have inspired this thruster concept and could in fact provide a proof of principle. The first qualitative experimental evidence of plasmoid formation demonstrated there was first predicted by global MHD simulations (Ebrahimi \& Raman 2015), later expanded for plasmoid-driven start-up in spherical tokamaks (Ebrahimi \& Raman 2016; Ebrahimi 2019). The extended MHD simulations presented here have been instrumental for exploring the fundamental physics of this new concept. However, more detailed physics (for example neutral dynamics and multi-fluid effects) could be numerically investigated in a future study to develop predictive capabilities for building a prototype device.

\section{Acknowledgements}

The author acknowledges insightful comments by J. Menard and S. Prager. Computations were performed at NERSC and local PPPL cluster. This work was supported by DOE grants DE-AC02-09CHI1466 and DE-SC0010565, and PPPL LDRD grant.

Editor William Dorland thanks the referees for their advice in evaluating this article.

\section{Declaration of interest}

The author reports no conflict of interest. 


\section{REFERENCES}

Alfven, H., Lindberg, L. \& Mitlid, P. 1960 Experiments with plasma rings. J. Nucl. Energy 1, 116-120.

Arefiev, A. V. \& Breizman, B. N. 2005 Magnetohydrodynamic scenario of plasma detachment in a magnetic nozzle. Phys. Plasmas 12 (4), 043504.

Bathgate, S. N., Bilek, M. M. M., Cairns, I. H. \& McKenzie, D. R. 2018 A thruster using magnetic reconnection to create a high-speed plasma jet. Eur. Phys. J.: Appl. Phys 84, 20801.

BAYliss, R. A., Sovinec, C. R. \& RedD, A. J. 2011 Zero- $\beta$ modeling of coaxial helicity injection in the HIT-II spherical torus. Phys. Plasmas 18 (9), 094502.

Bhattacharjee, A., Huang, Y.-M., Yang, H. \& Rogers, B. 2009 Fast reconnection in high-Lundquist-number plasmas due to the plasmoid Instability. J. Plasma Phys. 16, 112102.

BISKAMP, D. 1986 Magnetic reconnection via current sheets. Phys. Fluids 29, 1520-1531.

Brown, M. R. \& Bellan, P. M. 1990 Current drive by spheromak injection into a tokamak. Phys. Rev. Lett. 64, 2144-2147.

Cassak, P. A., Shay, M. A. \& Drake, J. F. 2005 Catastrophe model for fast magnetic reconnection onset. Phys. Rev. Lett. 95 (23), 235002.

Cheng, D. Y. 1971 Application of a deflagration plasma gun as a space propulsion thruster. AIAA J. 9, 1681-1685.

Chesny, D. L., Orange, N. B., Oluseyi, H. M. \& Valletta, D. R. 2017 Toward laboratory torsional spine magnetic reconnection. J. Plasma Phys. 83 (6), 905830602.

Choueiri, E. Y. 2009 New dawn for electric rockets. Sci. Am. 300 (2), 58-65.

Comisso, L., Lingam, M., Huang, Y.-M. \& Bhattacharjee, A. 2016 General theory of the plasmoid instability. Phys. Plasmas 23, 100702.

Dale, E., Jorns, B. \& Gallimore, A. 2020 Future directions for electric propulsion research. Aerospace 7 (9), 120.

Daughton, W., Roytershteyn, V., Albright, B. J., Karimabadi, H., Yin, L. \& Bowers, K. J. 2009 Transition from collisional to kinetic regimes in large-scale reconnection layers. Phys. Rev. Lett. 103 (6), 065004.

Ebrahimi, F. 2016 Dynamo-driven plasmoid formation from a current-sheet instability. Phys. Plasmas 23 (12), 120705.

Ebrahimi, F. 2017 Nonlinear reconnecting edge localized modes in current-carrying plasmas. Phys. Plasmas 24 (5), 056119.

Ebrahimi, F. 2019 Three-dimensional plasmoid-mediated reconnection and the effect of toroidal guide field in simulations of coaxial helicity injection. Phys. Plasmas 26 (9), 092502.

Ebrahimi, F., Hooper, E. B., Sovinec, C. R. \& Raman, R. 2013 Magnetic reconnection process in transient coaxial helicity injection. Phys. Plasmas 20, 090702.

Ebrahimi, F. \& Prager, S. C. 2011 Momentum transport from current-driven reconnection in astrophysical disks. Astrophys. J. 743, 192.

Ebrahimi, F., Raman, R., Hooper, E. B., Sovinec, C. R. \& Bhattacharjee, A. 2014 Physics of forced magnetic reconnection in coaxial helicity injection experiments in National Spherical Torus Experiment. J. Plasma Phys. 21, 056109.

EBRAHIMI, F. \& RAMAN, R. 2015 Plasmoids formation during simulations of coaxial helicity injection in the national spherical torus experiment. Phys. Rev. Lett. 114 (20), 205003.

EBRAHIMI, F. \& RAMAN, R. 2016 Large-volume flux closure during plasmoid-mediated reconnection in coaxial helicity injection. Nucl. Fusion 56 (4), 044002.

Fox, N. J., Velli, M. C., Bale, S. D., Decker, R., Driesman, A., Howard, R. A., Kasper, J. C., Kinnison, J., Kusterer, M., LARio, D., et al. 2016 The solar probe plus mission: humanity's first visit to our star. Space Sci. Rev. 204 (1-4), 7-48.

Goebel, D. M. \& Katz, I. 2008 Fundamentals of Electric Propulsion: Ion and Hall Thrusters, vol. 1. John Wiley \& Sons.

Günter, S., Yu, Q., Lackner, K., Bhattacharjee, A. \& Huang, Y.-M. 2015 Fast sawtooth reconnection at realistic Lundquist numbers. Plasma Phys. Control. Fusion 57, 014017. 
Hammer, J. H., Hartman, C. W., Eddleman, J. L. \& Mclean, H. S. 1988 Experimental demonstration of acceleration and focusing of magnetically confined plasma rings. Phys. Rev. Lett. 61, 2843-2846.

Hammond, K. C., Raman, R. \& Volpe, F. A. 2017 Application of townsend avalanche theory to tokamak startup by coaxial helicity injection. Nucl. Fusion 58 (1), 016013.

Hooper, E. B., Bulmer, R. H., Cohen, B. I., Hill, D. N., Holcomb, C. T., Hudson, B., Mclean, H. S., Pearlstein, L. D., Romero-Talamás, C. A., Sovinec, C. R., et al. 2012 Sustained spheromak physics experiment (SSPX): design and physics results. Plasma Phys. Control. Fusion 54 (11), 113001.

Hooper, E. B., Sovinec, C. R., Raman, R., Ebrahimi, F. \& Menard, J. E. 2013 Resistive magnetohydrodynamic simulations of helicity-injected startup plasmas in national spherical torus experiment. Phys. Plasmas. 20, 092510.

Hsu, S. C. \& Bellan, P. M. 2003 Experimental identification of the kink instability as a poloidal flux amplification mechanism for coaxial gun spheromak formation. Phys. Rev. Lett. 90 (21), 215002.

Huang, Y.-M., Bhattacharjee, A. \& Sullivan, B. P. 2011 Onset of fast reconnection in hall magnetohydrodynamics mediated by the plasmoid instability. Phys. Plasmas 18 (7), 072109.

Jarboe, T. R., Henins, I., Sherwood, A. R., Barnes, C. W. \& Hoida, H. W. 1983 Slow formation and sustainment of spheromaks by a coaxial magnetized plasma source. Phys. Rev. Lett. 51, 39-42.

Ji, H., Alt, A., Antiochos, S., BaAlrud, S., Bale, S., Bellan, P. M., Begelman, M., Beresnyak, A., Blackman, E. G., Brennan, D., et al. 2020 Major scientific challenges and opportunities in understanding magnetic reconnection and related explosive phenomena throughout the universe. arXiv:2004.00079.

Ji, H. \& Daughton, W. 2011 Phase diagram for magnetic reconnection in heliophysical, astrophysical, and laboratory plasmas. Phys. Plasmas 18 (11), 111207.

Loureiro, N. F., Schekochinin, A. A. \& Cowley, S. C. 2007 Instability of current sheets and formation of plasmoid chains. Phys. Plasmas 14 (10), 100703.

Lovberg, R. H. 1971 16. Plasma problems in electrical propulsion. In Methods in Experimental Physics, vol. 9, pp. 251-289. Elsevier.

Marshall, J. 1960 Performance of a hydromagnetic plasma gun. Phys. Fluids 3, 134-135.

Mclean, H. S., Woodruff, S., Hooper, E. B., Bulmer, R. H., Hill, D. N., Holcomb, C., Moller, J., Stallard, B. W., Wood, R. D. \& WAng, Z. 2002 Suppression of MHD fluctuations leading to improved confinement in a gun-driven spheromak. Phys. Rev. Lett. 88 (12), 125004.

Morgan, K., JARBOE, T. \& AKCAY, C. 2019 Formation of closed flux surfaces in spheromaks sustained by steady inductive helicity injection. Nucl. Fusion 59 (6), 066037.

Morozov, A. I., Esipchuk, Y. V., Kapulkin, A. M., Nevrovskit, V. A. \& Smirnov, V. A. 1972 Effect of the magnetic field a closed-electron-drift accelerator. Sov. Phys. Tech. Phys. 17, 482.

Raitses, Y., SMirnov, A. \& Fisch, N. J. 2007 Enhanced performance of cylindrical hall thrusters. Appl. Phys. Lett. 90 (22), 221502.

Raman, R., Jarboe, T. R., Nelson, B. A., Izzo, V. A., O’Neill, R. G., Redd, A. J. \& Smith, R. J. 2003 Demonstration of plasma startup by coaxial helicity injection. Phys. Rev. Lett. 90 (7), 075005.

Raman, R., Martin, F., Quirion, B., St-Onge, M., Lachambre, J.-L., Michaud, D., Sawatzky, B., Thomas, J., Hirose, A., Hwang, D., et al. 1994 Experimental demonstration of nondisruptive, central fueling of a tokamak by compact toroid injection. Phys. Rev. Lett. 73, 3101-3104.

Raman, R., Mueller, D., Jarboe, T. R., Nelson, B. A., Bell, M. G., Gerhardt, S., LeBlanc, B., Menard, J., Ono, M., Roquemore, L., et al. 2011 Experimental demonstration of tokamak inductive flux saving by transient coaxial helicity injection on national spherical torus experiment. Phys. Plasmas 18 (9), 092504.

RipPERDA, B., BACCHINI, F. \& PHILIPPOV, A. 2020 Magnetic reconnection and hot-spot formation in black-hole accretion disks. arXiv:2003.04330.

Schoenberg, K. F., Gerwin, R. A., Henins, I., Mayo, R. M., Scheuer, J. T. \& Wurden, G. A. 1993 Preliminary investigation of power flow and performance phenomena in a multimegawatt coaxial plasma thruster. IEEE Trans. Plasma Sci. 21, 625-644. 
Sovinec, C. R., Glasser, A. H., Gianakon, T. A., Barnes, D. C., Nebel, R. A., Kruger, S. E., Schnack, D. D., Plimpton, S. J., TARditi, A., Chu, M., et al. 2004 Nonlinear magnetohydrodynamics simulation using high-order finite elements. J. Comput. Phys. 195, 355.

StUhlinger, E. 1964 Ion Propulsion for Space Flight. McGraw-Hill.

TAJima, T. \& Shibata, K. (Ed.) 1997 Plasma Astrophysics. Addison-Wesley.

Witherspoon, F. D., CAse, A., Messer, S. J., Bomgardner, R., Phillips, M. W., BRockington, S. \& Elton, R. 2009 A contoured gap coaxial plasma gun with injected plasma armature. Rev. Sci. Instrum. 80 (8), 083506.

Yoo, J., Na, B., Jara-Almonte, J., Yamada, M., Ji, H., Roytershteyn, V., Argall, M. R., FoX, W. \& CHEN, L.-J. 2017 Electron heating and energy inventory during asymmetric reconnection in a laboratory plasma. J. Geophys. Res.: Space Phys. 122 (9), 9264-9281.

ZWeibel, E. G. \& YAMADA, M. 2009 Magnetic reconnection in astrophysical and laboratory plasmas. Annu. Rev. Astron. Astrophys. 47, 291-332. 